\title{
A qualidade das refeições de empresas cadastradas no Programa de Alimentação do Trabalhador na cidade de São Paulo'
}

\section{The quality of meals in companies participating in the worker's food program in the city of São Paulo, Brazil}

Daniel Henrique BANDONI ${ }^{2}$

Patrícia Constante JAIME²

RE S U M O

\section{Objetivo}

Avaliar a qualidade global das refeições oferecidas por Unidades de Alimentação e Nutrição de empresas beneficiárias do Programa de Alimentação do Trabalhador, na cidade de São Paulo.

\section{Métodos}

Estudo transversal realizado com 72 empresas cadastradas no programa. Foram coletadas informações de três dias consecutivos das refeições oferecidas no almoço, no jantar e na ceia. A qualidade das refeições oferecidas foi avalia pelo Índice de Qualidade da Refeição, e sua análise foi feita de forma estratificada segundo o perfil da empresa obtido pela análise de cluster.

\section{Resultados}

A média do Índice de Qualidade da Refeição para as grandes refeições foi de 66,25. Foram obtidos dois grupos de empresas na análise de cluster. As empresas do primeiro, composto em sua maioria por empresas do setor de comércio de micro e pequeno porte, cadastradas na modalidade de autogestão e sem supervisão de nutricionista, obtiveram pior qualidade da refeição (Índice=56,23). As empresas do segundo cluster, constituído principalmente por empresas de médio e grande porte do setor industrial, com gestão terceirizada e supervisão de nutricionista, obtiveram pontuação média do Índice de 82,95.

\section{Conclusão}

As refeições oferecidas pelas empresas participantes do Programa de Alimentação do Trabalhador não estavam adequadas, segundo o Índice de Qualidade da Refeição. As empresas de menor porte e estrutura tiveram

\footnotetext{
1 Artigo elaborado a partir da dissertação de D.H. BANDONI, intitulada "Índice de qualidade da refeição de empresas cadastradas no Programa de Alimentação do Trabalhador da cidade de São Paulo". Universidade de São Paulo; 2006; Apoio: Conselho Nacional de Desenvolvimento Científico e Tecnológico, processo no 471136/03-4.

2 Universidade de São Paulo, Faculdade de Saúde Pública, Departamento de Nutrição. Av. Dr Arnaldo, 715, 01246-904, São Paulo, SP, Brasil. Correspondência para/Correspondence to: D.H. BANDONI. E-mail: <dbandoni@usp.br>.
} 
178 D.H. BANDONI \& P.C. JAIME

refeições de pior qualidade quando comparadas com as demais, demonstrando que empresas deste perfil são prioritárias para intervenções dentro do Programa de Alimentação do Trabalhador.

Termos de indexação: Alimentação Coletiva. Local de trabalho. Programas e Políticas de Nutrição e Alimentação. Serviços de Alimentação.

\section{A B S T R A C T}

\section{Objective}

The aim of this study was to assess the global quality of the meals offered in the Food and Nutrition Units of companies participating in the Worker's Food Program, in the city of São Paulo.

\section{Methods}

This was a cross-sectional study of 72 companies participating in the program. Information regarding three consecutive days of meals served during lunch, supper and late supper were collected. The quality of the meals was assessed by the Meals Quality Index and its analysis was done in a stratified manner according to the company profile obtained through cluster analysis.

\section{Results}

The Meals Quality Index mean for the meals was 66.25\%. Two groups of companies were obtained through cluster analysis. The companies in the first group consisted mostly of stores and micro- and small-sized companies, registered in the category of self-management and without the supervision of a dietician. They presented the worst meal quality (Index $=56.23$ ). The companies in the second cluster consisted mostly of medium- and big-sized companies that outsourced meal production and counted with the supervision of a dietician. Their mean score on the Index was 82.95.

\section{Conclusion}

The meals offered by the companies participating in the Worker's Food Program were not adequate according to the Meals Quality Index. The smaller companies in size and structure had worse meals when compared with the other companies, showing that companies with this profile should be the priority of interventions within the Worker's Food Program.

Indexing terms: Collective feeding. Workplace. Nutrition programmes and policies. Food services.

\section{N T R O D U ÇÃ O}

O Programa de Alimentação do Trabalhador (PAT) é uma das mais antigas e importantes políticas de alimentação e nutrição do Brasil. Com inquestionável relevância social, é considerado um herdeiro de políticas públicas nacionais anteriores voltadas à alimentação do trabalhador ${ }^{1}$.

O PAT vem atraindo cada vez mais interesse para a promoção de estilos de vida saudáveis, pois, com o atual quadro epidemiológico, de avanço das doenças crônicas e da obesidade, o ambiente de trabalho vem sendo reconhecido como um local propício às modificações de comportamento precursor de doenças, não apenas associados à função ocupacional mas também à dieta, à atividade física e ao tabagismo².
A "Estratégia Global sobre Alimentação, Atividade Física e Saúde", da Organização Mundial da Saúde, considera as empresas que fornecem alimentação coletiva como protagonistas importantes na promoção de uma alimentação saudável. As iniciativas devem voltar-se à redução da quantidade de gorduras, açúcar e sal dos alimentos, elaborando opções inovadoras e saudáveis nos cardápios, além de promover o consumo de frutas, legumes e verduras ${ }^{3}$.

Contudo, estudos prévios vêm demonstrando inadequação das refeições oferecidas pelo programa, com excesso de proteínas e gorduras e baixas quantidades de fibras, frutas e hortaliças ${ }^{4-6}$. Estudos também têm encontrado uma alta prevalência de excesso de peso entre os traba- 
Ihadores beneficiados, demonstrando que o PAT pode ter um efeito negativo sobre o estado nutricional dos trabalhadores ${ }^{5,7}$.

Entretanto, são poucos os estudos que avaliam a qualidade das refeições oferecidas nas empresas beneficiárias pelo PAT. Assim, este trabalho tem como objetivo avaliar a qualidade global das refeições oferecidas por Unidades de Alimentação e Nutrição de empresas beneficiárias do Programa de Alimentação do Trabalhador na cidade de São Paulo.

\section{MÉ T O D OS}

Trata-se de um estudo observacional transversal, com amostra aleatória de 93 empresas inscritas no PAT, localizadas na cidade de São Paulo. O tamanho da amostra corresponde a $12,9 \%$ das empresas cadastradas no ano de 2003, inscritas nas modalidades autogestão e gestão terceirizada (preparo e distribuição de refeição e refeição transportada).

O banco de dados das empresas inscritas no PAT na cidade de São Paulo, utilizado no sorteio da amostra, foi fornecido pela Coordenação Geral do programa do Ministério do Trabalho e Emprego. A coleta de dados foi realizada por entrevistadores devidamente treinados, utilizando questionários padronizados, e ocorreu entre outubro de 2003 e março de 2004. Ao final desta etapa houve um total de 21 perdas (22,0\%), sendo 11 recusas e 10 empresas que não foram encontradas, sendo investigadas 72 empresas.

Avaliaram-se as grandes refeições (almoço, jantar e ceia) oferecidas aos trabalhadores durante três dias, utilizando-se os per capitas dos alimentos e preparações fornecidas pelas empresas aos trabalhadores.

As quantidades per capita foram estabelecidas por meio de consulta ao receituário padrão das Unidades de Alimentação e Nutrição, considerando todos os alimentos oferecidos, independentemente do tipo de serviço adotado pelas empresas. Apenas para as unidades que não possuíam o receituário, calculou-se o per capita dividindo-se a quantidade de alimento utilizada, obtida a partir do registro da saída de estoque, pelo número total de refeições servidas no dia. As quantidades per capita também foram corrigidas com o indicador de partes não comestíveis determinadas no Estudo Nacional de Despesa Familiar (ENDEF), 1974-1975

Para o cálculo do valor nutricional das refeições foi utilizada, inicialmente, a Tabela Brasileira de Composição de Alimentos (TACO) ${ }^{9}$, e adicionalmente dados da tabela de composição do Departamento de Agricultura dos Estados Unidos (USDA), versões 16 e $17^{10}$.

Para avaliar a qualidade global das refeições oferecidas pelo PAT, utilizou-se o Índice de Qualidade da Refeição (IQR), que propõe a utilização de 5 variáveis, as quais recebem pontuação de 0 a 20, considerando simultaneamente a oferta de alimentos e nutrientes, permitindo uma avaliação indireta de componentes da refeição sem reduzir a avaliação a um único item. A distribuição entre os valores 0 e 20 é feita de forma proporcional, assim, conforme o componente está mais próximo do adequado, maior será a sua pontuação. Os componentes do índice são os seguintes:

1. Adequação na oferta de verduras, legumes e frutas: foram verificadas a adequação das quantidades em gramas por refeição, sendo que uma oferta de $160 \mathrm{~g}$ ou mais recebeu pontuação 20 e a oferta igual ou inferior a $80 \mathrm{~g}$ recebeu pontuação igual a 0;

2. Oferta de carboidratos: oferta percentual em relação à energia, considerando uma oferta ideal entre $55 \%$ e $75 \%$ do total de calorias; que equivalia à pontuação 20 . A oferta inferior a $40 \%$ recebeu pontuação igual a 0 . Não houve nenhuma refeição com oferta de carboidratos acima de $75 \%$;

3. Oferta de gordura total: oferta percentual em relação à energia considerando uma oferta ideal entre $15 \%$ e $30 \%$ do total de calorias, que recebeu pontuação 20 e a oferta superior a 
$40 \%$, que recebeu pontuação igual a 0, nenhuma empresa ofereceu menos que $15 \%$ de gorduras;

4. Oferta de gordura saturada: oferta percentual em relação à energia considerando o total de energia proveniente dos ácidos graxos saturados menor que $10 \%$, que recebeu pontuação 20 e a oferta superior a $13 \%$, que recebeu pontuação igual a 0;

5. Variabilidade da refeição: indicador considerou o número de alimentos (pontuação de 0 a 7) e o número de grupos de alimentos (pontuação de 0 a 3), somando os pontos obtidos nestes dois indicadores. Assim, a refeição que oferecia no mínimo 11 diferentes alimentos e 5 diferentes grupos de alimentos recebia 20, enquanto que a refeição que oferecesse menos de dois grupos e 4 alimentos recebia 0.

O IQR foi previamente desenvolvido a partir de uma análise de 10 indicadores, para obter a melhor composição do índice que avaliasse de forma global as refeições. Utilizou-se o teste do Alfa de Cronbach, teste utilizado para avaliar a consistência interna do indicador ${ }^{11}$.

Calculou-se a média do IQR do almoço, do jantar e da ceia, para a definição do IQR das grandes refeições oferecidas no PAT, que constituem as principais refeições oferecidas pelas empresas beneficiárias do programa. Classificouse o IQR de acordo com a proposta de Bowman et al. ${ }^{12}$, que considera como adequada aquela refeição que obtiver pontuação maior que 80; refeição que precisa de melhoras com a pontuação entre 51 e 80 e refeição inadequada com pontuação menor ou igual a 50 .

As empresas foram caracterizadas de acordo com o setor de atividade econômica, porte da empresa, modalidade de adesão ao PAT e supervisão de nutricionista. Para o setor de atividade econômica as empresas foram classificadas em industrial, serviços ou comércio, segundo seu registro no cadastro do PAT no Ministério do Trabalho e Emprego. Para o porte da empresa, essas foram classificadas em micro, pequeno, médio e grande porte, de acordo com o número de funcionários adotando a classificação utilizada por Moura ${ }^{13}$, e a proposta do Serviço brasileiro de apoio às micro e pequenas empresas ${ }^{14}$ para micro e pequenas empresas.

Na modalidade de adesão ao PAT considerou-se: autogestão quando a empresa beneficiária assume toda a responsabilidade pela elaboração das refeições; gestão terceirizada do tipo refeição transportada, quando a refeição foi preparada em cozinha industrial e transportada até o local de trabalho; e a gestão terceirizada do tipo preparo e distribuição de refeição na empresa, quando a empresa contrata os serviços de uma terceira, que utiliza as suas instalações para o preparo e a distribuição das refeições. Para supervisão de nutricionista, classificou-se como sim ou não, de acordo com a atuação de um responsável técnico no local.

Foi realizada uma análise descritiva dos dados por meio de freqüências e medidas de tendência central (médias, desvios-padrão (DP) e valores mínimos e máximos), para caracterização das refeições e das empresas.

Comparou-se a média do IQR entre o grupo das empresas utilizando-se o teste " $\mathrm{t}$ "-Student para amostras independentes. Realizou-se análise de cluster (agrupamento) hierárquico com as variáveis: setor de atividade econômica, porte da empresa, modalidade de adesão ao programa e supervisão de nutricionista. Optou-se pela análise de cluster, pois estas variáveis apresentaram elevada associação, possuindo características semeIhantes, que comprometem a análise isolada de cada uma delas. A formação do cluster foi realizada a partir da análise do dendograma, que representa o agrupamento das empresas pelas características selecionadas, identificando dois diferentes grupos (cluster 1 e 2).

Foi utilizado o programa estatístico SPSS (versão 11.0; SPSS Inc, Chicago) para elaboração do banco de dados e sua análise, considerando um nível de significância de 5\%.

Este estudo foi feito de acordo com a resolução 196/96 do Conselho Nacional de Saúde e 
aprovado pelo comitê de ética da Faculdade de Saúde Pública da Universidade de São Paulo (Protocolo n.1145).

\section{RES U LTADOS}

Foram avaliadas 72 empresas inscritas no PAT na cidade de São Paulo, que beneficiam mais de 20 mil trabalhadores com refeição. Na descrição das características das empresas (Tabela 1), observou-se que maioria pertence ao setor de comércio $(41,7 \%)$, com serviço de alimentação de autogestão (61,1\%). Na análise dos dois grupos, observa-se que o cluster 1 tem, na sua maioria, empresas de menor porte do setor de comércio, com autogestão e sem supervisão de nutricionista, o oposto do que ocorre no cluster 2 .

A média do Índice para as grandes refeições foi de 66,25 pontos. Na análise dos componentes e do índice médio das grandes refeições (Tabela 2), segundo os clusters, observou-se que a pontuação média do cluster 2 foi 26,72 pontos maior que o cluster 1 , diferença estatisticamente significante. As principais diferenças entre os dois grupos foram nos componentes de frutas e hortaliças, gorduras saturadas e variedade da refeição.

Quando é realizada a classificação do IQR, observa-se que no cluster 1 a maioria das empresas teve a refeição classificada com precisa de melhoras, enquanto que no cluster 2 a maioria das empresas fornece refeições adequadas (Tabela 3), ressaltando, assim, a diferença na qualidade das refeições oferecidas entre os dois grupos.
Tabela 1. Características das empresas inscritas no Programa de Alimentação do Trabalhador e composição dos clusters. São Paulo (SP), 2004.

\begin{tabular}{|c|c|c|c|c|c|c|}
\hline \multirow{2}{*}{$\begin{array}{l}\text { Características das } \\
\text { empresas }\end{array}$} & \multicolumn{2}{|c|}{ Cluster 1} & \multicolumn{2}{|c|}{ Cluster 2} & \multicolumn{2}{|c|}{ Total } \\
\hline & $n$ & $\%$ & $n$ & $\%$ & $n$ & $\%$ \\
\hline \multicolumn{7}{|l|}{ Setor de Atividade } \\
\hline Industrial & 2 & 9,5 & 19 & 90,5 & 21 & 29,2 \\
\hline Serviços & 13 & 61,9 & 8 & 38,1 & 21 & 29,2 \\
\hline Comércio & 30 & 100,0 & 0 & 0 & 30 & 41,7 \\
\hline \multicolumn{7}{|l|}{ Porte da Empresa } \\
\hline Micro e pequena & 33 & 94,3 & 2 & 5,7 & 35 & 48,6 \\
\hline Média e grande & 12 & 32,4 & 25 & 67,6 & 37 & 51,4 \\
\hline \multicolumn{7}{|l|}{$\begin{array}{l}\text { Modalidade de } \\
\text { adesão }\end{array}$} \\
\hline Autogestão & 40 & 90,9 & 4 & 9,1 & 44 & 61,1 \\
\hline Gestão terceirizada & 5 & 17,9 & 23 & 82,1 & 28 & 38,9 \\
\hline \multicolumn{7}{|l|}{$\begin{array}{l}\text { Supervisão do } \\
\text { nutricionista }\end{array}$} \\
\hline Sim & 18 & 40,9 & 26 & 59,1 & 44 & 61,1 \\
\hline Não & 27 & 96,4 & 1 & 3,6 & 28 & 61,1 \\
\hline Total & 45 & 62,5 & 27 & 37,5 & 72 & 100,0 \\
\hline
\end{tabular}

Tabela 3. Classificação do Índice de Qualidade da Refeição (IQR) das empresas inscritas no Programa de Alimentação do Trabalhador, de acordo com os clusters. São Paulo (SP), 2004

\begin{tabular}{|c|c|c|c|c|c|c|}
\hline \multirow{2}{*}{ Classificação do IQR } & \multicolumn{2}{|c|}{ Cluster 1} & \multicolumn{2}{|c|}{ Cluster 2} & \multicolumn{2}{|c|}{ Total } \\
\hline & $n$ & $\%$ & $\mathrm{n}$ & $\%$ & $\mathrm{n}$ & $\%$ \\
\hline Refeição inadequada & 12 & 26,7 & 3 & 11,1 & 15 & 20,8 \\
\hline $\begin{array}{l}\text { Refeição que precisa de } \\
\text { melhoras }\end{array}$ & 30 & 66,7 & 8 & 29,6 & 38 & 52,7 \\
\hline Refeição adequada & 3 & 6,7 & 16 & 59,6 & 19 & 26,5 \\
\hline
\end{tabular}

Tabela 2. Pontuação média do índice de qualidade (IQR) da Refeição e dos seus componentes, das refeições oferecidas pelo Programa de Alimentação do Trabalhador, segundo cluster. São Paulo (SP), 2004.

\begin{tabular}{|c|c|c|c|c|c|c|}
\hline \multirow{2}{*}{ Classificação do IQR } & \multicolumn{2}{|c|}{ Cluster 1} & \multicolumn{2}{|c|}{ Cluster 2} & \multicolumn{2}{|c|}{ Total } \\
\hline & M & DP & M & DP & M & DP \\
\hline Indicador de frutas e hortaliças ${ }^{1}$ & 5,77 & 7,59 & 17,42 & 4,82 & 10,14 & 8,74 \\
\hline Indicador de carboidratos & 12,69 & 7,17 & 14,75 & 6,76 & 13,46 & 7,04 \\
\hline Indicador de gorduras & 13,58 & 7,71 & 15,71 & 6,63 & 14,26 & 7,33 \\
\hline Indicador de gorduras saturadas ${ }^{1}$ & 14,70 & 7,58 & 19,19 & 2,58 & 16,38 & 6,54 \\
\hline Indicador de variedade ${ }^{1}$ & 9,50 & 6,64 & 18,02 & 3,37 & 12,69 & 6,99 \\
\hline Índice de Qualidade da Refeição ${ }^{1}$ & 56,23 & 21,35 & 82,95 & 17,78 & 66,25 & 23,83 \\
\hline
\end{tabular}

1 Diferença significante no teste " $t$ " ( $p<0,001)$; M: média; DP: desvio-padrão. 


\section{DISCUSSÃ O}

Este trabalho possíbilitou avaliar a qualidade global das refeições oferecidas em empresas participantes do Programa de Alimentação do Trabalhador (PAT), segundo suas principais características. Na análise de cluster obteve-se dois diferentes grupos, o primeiro caracterizado por empresas de menor porte do setor de serviços e comércio e com modalidade de autogestão. Já o segundo tem, em sua maioria, empresas de maior porte, do setor industrial, e com gestão terceirizada. Observou-se que, no primeiro cluster, a maioria das empresas não tem supervisão do nutricionista, demonstrando que empresas de menor porte têm dificuldade em contratar e manter um responsável técnico, que é exigência da legislação vigente do programa.

A média do índice para as grandes refeições foi classificada como refeição que precisa de melhoras, demonstrando que as refeições servidas no PAT não estão totalmente adequadas às recomendações mais recentes para alimentação saudável.

É interessante observar que a média do IQR ficou próxima ao achado de Fisberg ${ }^{15}$, que encontrou média de 60, 42 pontos no Índice de Qualidade da Dieta de indivíduos adultos residentes na região metropolitana de São Paulo. Demonstra-se, assim, que a qualidade das refeições do programa não difere muito da alimentação consumida na região da cidade de São Paulo, apesar de as comparações serem limitadas pelas diferenças nos instrumentos.

Estudos recentes demonstraram a inadequação das refeições oferecidas no programa. Entre os principais pontos se destacavam os excessos de gorduras totais e a baixa quantidade de fibras e carboidratos 6 ,13,14,16. Esses resultados reforçam a importância de aumentar a oferta de frutas e hortaliças em refeições oferecidas por empresas pertencentes ao programa, que levaria a um aumento na variedade do cardápio e nas fibras, podendo ajudar a reduzir a densidade energética das refeições.
Diferentemente desses estudos, no presente trabalho os indicadores de gorduras e gorduras saturadas obtiveram um bom desempenho. Assim, observa-se que nesta amostra as quantidades de gorduras, principalmente as saturadas, estavam mais próximas do recomendado. Este resultado pode ser explicado pela diferença metodológica na forma de coletar as quantidades oferecidas pelas refeições, que procurou determinar as quantidades de alimentos utilizadas para o preparo. Apesar de terem sido consideradas as quantidades que retornavam ao estoque ou que não eram utilizadas durante a elaboração das refeições, não se avaliou o que foi consumido exatamente pelo trabalhador, isso poderia levar à diminuição na ingestão de alguns alimentos e aumentar a participação das gorduras no total energético. Além disso, alguns estudos definiram como percentual máximo de gordura $25 \%$ da participação energética total, diferente do presente estudo que considerou $30 \%$.

Com os resultados obtidos, fica reforçada a idéia de que o programa pode estar colaborando para o desequilíbrio nutricional da população beneficiada. Trabalho de Veloso \& Santana ${ }^{7}$ encontrou associação entre o PAT na Bahia e o aumento de peso dos trabalhadores. Savio et al. ${ }^{5}$ encontraram alta prevalência de excesso de peso em trabalhadores beneficiados pelo programa, principalmente entre os homens.

Ao analisar a média do Índice para as grandes refeições, segundo as características das empresas, observa-se que o cluster 2 tem uma melhor qualidade da refeição. A diferença de pontuação entre as empresas predominantemente de menor porte, do setor de comércio e serviços, inscritas na modalidade de autogestão e sem supervisão de nutricionista, é de 26,72 pontos. As principais diferenças estão nos indicadores de frutas e hortaliças, gorduras saturadas e variedade, demonstrando que estas empresas oferecem cardápios com menor variedade, mais restritos e com maiores quantidades de gordura animal.

Alguns fatores podem contribuir para que as empresas de menor estrutura tenham uma 
qualidade inferior da refeição, tais como: o menor número de funcionários beneficiados que leva à preparação de refeições mais simples, diminuindo a oferta de frutas e hortaliças; e a ausência de um nutricionista, responsável técnico, que possa elaborar cardápios adequados aos trabalhadores.

Este resultado demonstra que o perfil da empresa é um determinante da qualidade da refeição que será oferecida aos trabalhadores. Assim, quanto menor for a estrutura para fornecer alimentação, pior será a qualidade da refeição oferecida. Esta informação é importante para o aperfeiçoamento do programa, já que um dos pontos mais debatidos é o aumento da participação de micro e pequenas empresas no PAT ${ }^{17}$.

Uma das limitações do presente trabalho, foi ter avaliado apenas as empresas que ofereciam refeição no local de trabalho, o que excluiu modalidades representativas, como a refeição convênio.

Contudo, se a expansão do programa para estas empresas for feita sem a conscientização e orientação destas e de seus gestores, aparentemente, pode levar a uma oferta de refeições desequilibradas para os trabalhadores. Estudo realizado com os gestores do PAT, nesta mesma amostra, demonstrou que a maioria desconhecia os objetivos do programa de promoção de saúde por meio da alimentação ${ }^{18}$.

Certamente, o PAT tem grande importância nas políticas de alimentação e nutrição do Brasil, sendo o único voltado para a população trabalhadora. Em agosto de 2006, após 30 anos de criação do programa, foi publicada portaria que altera as exigências nutricionais do PAT, modificando as recomendações de energia e adicionando recomendações para macronutrientes, gorduras saturadas, fibras e sódio, que não eram contempladas anteriormente. Além disso, a portaria também acrescenta a obrigatoriedade da oferta de frutas, verduras e legumes nas refeições oferecidas pelas empresas e se ajusta às recomendações do guia alimentar para a população brasileira.
Os achados deste estudo apontam para a necessidade de orientar, prioritariamente, as empresas menores para que elas ajustem seus cardápios aos novos parâmetros nutricionais do programa, conheçam os objetivos do PAT e saibam como os alcançar, já que sua participação no programa é fundamental para sua ampliação e aumento da cobertura de trabalhadores, principalmente de baixa renda.

De tal modo, é fundamental a utilização de instrumentos adequados de avaliação das refeições oferecidas, para que o PAT se consolide como uma política de promoção de alimentação saudável e saúde, indispensável no Brasil.

\section{COLABORADORES}

D.H. BANDONI e P.C. JAIME participaram da coleta de dados, da tabulação, da discussão dos resultados e da elaboração do artigo.

\section{REFERÊ NCIAS}

1. Schmitz BAS, Heyde MEDVD, Cintra IP, Franceschini SCC, Taddei JAAC, Sigulem DM. Políticas e programas governamentais de alimentação e nutrição no Brasil e sua involução. Cad Nutr. 1997; 13(1):39-54

2. Chu C, Breucker G, Harris N, Stitzel A, Xinga G, Gu $X$, et al. Health promoting workplaces - interational settings development. Health Promot Int. 2000; 15(2):155-67.

3. World Health Organization. Global Strategy on diet, physical activity and health. Food Nutr Bull. 2004; 25(3):292-302.

4. Jaime PC, Bandoni DH, Geraldo APG, Rocha RV. Adequação das refeições oferecidas por empresas cadastradas no programa de alimentação do trabalhador na cidade de São Paulo. Mundo da Saúde. 2005; 29(2):186-91.

5. Savio KEO, Costa THM, Miazaki E, Schmitz BAS. Avaliação do almoço servido a participantes do programa de alimentação do trabalhador. Rev Saúde Pública. 2005; 39(2):145-55.

6. Freire RBM, Salgado RS. Avaliação de cardápios oferecidos a trabalhadores horistas. Mundo Saúde. 1995; 22(5):298-301.

7. Veloso IS, Santana VS. Impacto nutricional do programa de alimentação do trabalhador no Brasil. Rev Panam Salud Publica. 2002; 11(1):24-31. 
8. Instituto Brasileiro de Geografia e Estatística. Estudo Nacional de Despesas Familiares - ENDEF, 1974-1975. Rio de Janeiro; 1978.

9. Universidade de Campinas. Tabela de composição de alimentos. [acesso 2005 jul 10]. Disponível em: $<$ http://www.unicamp.br/nepa/taco/>.

10. United States Departament of Agriculture. Nutrient Data Laboratory. [cited 2005 Jul 25]. Available from: <http://www.nal.usda.gov/fnic/foodcomp/>.

11. Bandoni, DH. Índice de qualidade da refeição de empresas cadastradas no programa de alimentação do trabalhador da cidade de São Paulo [dissertação]. São Paulo: Universidade de São Paulo; 2006.

12. Bowman AS, Lino M, Gerrior AS, Basiotis PP. The healthy eating index: 1994-1996. Washington (DC): Departament of Agriculture, Center for Nutrition Policy and Promotion; 1998.

13. Moura JB. Avaliação do programa de alimentação do trabalhador no estado de Pernambuco, Brasil. Rev Saúde Pública. 1986; 20(2):115-28.

14. Serviço Brasileiro de Apoio às Micro e Pequenas Empresas. Legislação Básica da micro e pequena empresa. [acesso 2004 jul 10]. Disponível em: <http://www.sebrae.com.br/br/aprendasebrae/ estudosepesquisas.asp>.

15. Fisberg RM, Slater B, Barros RR, Lima FD, César $C L G$, Carandina $L$, et al. Índice de qualidade da dieta: avaliação da adaptação e aplicabilidade. Rev Nutr. 2004; 17(3):301-8.

16. Gambardella AMD. O programa de alimentação do trabalhador frente às recomendações nutricionais para esse segmento específico da população: área metropolitana de São Paulo [dissertação]. São Paulo: Universidade de São Paulo; 1990.

17. Colares LGT. Evolução e perspectivas do programa de alimentação do trabalhador no contexto político brasileiro. Nutrire. 2005; 29(1):141-58.

18. Bandoni DH, Brasil BG, JAIME PC. Programa de alimentação do trabalhador: representações sociais de gestores locais. Rev Saúde Pública. 2006; 40(5): 837-42.

Recebido em: 11/5/2007

Versão final reapresentada em: 27/2/2008 Aprovado em: 27/2/2008 